\title{
Dimensionalidade da Escala de Estresse Percebido (Perceived Stress Scale, PSS-10) em uma Amostra de Professores
}

\section{Dimensionality of the Perceived Stress Scale (PSS-10) for School Teachers}

\author{
Wagner de Lara Machado, ${ }^{*}$, Bruno Figueiredo Damásio ${ }^{b}$, Juliane Callegaro Borsa $^{c}$ \\ \& Joilson Pereira da Silva ${ }^{d}$ \\ ${ }^{a}$ Universidade Federal do Rio Grande do Sul, Porto Alegre, Rio Grande do Sul, Brasil, \\ ${ }^{b}$ Universidade Federal do Rio de Janeiro, Rio de Janeiro, Rio de Janeiro, Brasil \\ cPontifícia Universidade Católica do Rio de Janeiro, Rio de Janeiro, Rio de Janeiro, Brasil \\ $\&{ }^{d}$ Universidade Federal de Sergipe, Itabaiana, Sergipe, Brasil
}

\begin{abstract}
Resumo
O presente estudo teve por objetivo investigar a dimensionalidade da Escala de Estresse Percebido em sua versão de 10 itens (PSS-10) em uma amostra de professores $(N=517)$. Uma análise fatorial exploratória pelo método de extração minimum rank factor analysis, utilizando a matriz de correlações policóricas e o critério de análise paralela para retenção dos fatores indicou a solução unifatorial como a mais ajustada aos dados. Os itens possuíram cargas fatoriais adequadas e o fator retido explicou $67 \%$ da variância comum dos escores, apresentando consistência interna $(\alpha=0,80)$. Este estudo fornece evidências de validade da PSS-10 no contexto brasileiro.

Palavras-chave: Escala de Estresse Percebido, PSS-10, análise fatorial exploratória, análise paralela, métodos robustos.
\end{abstract}

\begin{abstract}
The present study aimed to investigate the dimensionality of the 10-item Perceived Stress Scale (PSS10) in a sample of school teachers $(N=517)$. An exploratory factor analysis done by the extraction method of minimum rank factor analysis, using polychoric correlation matrix and parallel analysis criterion for factor retention, indicated a one-factor solution as the best fit to the data. The items showed adequate factor loadings and the retained factor explained $67 \%$ of common scores variance, presenting internal consistency $(\alpha=.80)$. This study provides validity evidences of the PSS-10 for the Brazilian context.

Keywords: Perceived Stress Scale, PSS-10, exploratory factor analysis, parallel analysis, robust methods.
\end{abstract}

A docência do ensino fundamental e médio é, no Brasil, considerada como uma das atividades profissionais mais estressantes, entre todas as categorias profissionais (Kyriacou, 2003). De acordo com um levantamento nacional realizado por Codo (1999), 26\% dos professores nacionais apresentavam um nível de exaustão emocional considerado perigoso à saúde mental dos mesmos. Esta não é uma realidade apenas brasileira. Diversos estudos internacionais tem, da mesma forma, demonstrado que as condições adversas da docência escolar são múltiplas, e contribuem para altos índices de estresse ocupacional e burnout (Doménech Betoret \& Gómez Artiga, 2010; Kerr, Breen, Delaney, Kelly, \& Miller, 2011; Kokkinos, 2007; Montgomery \& Rupp, 2005; Yong \& Yue, 2007).

* Endereço para correspondência: Instituto de Psicologia Universidade Federal do Rio Grande do Sul, Rua Ramiro Barcelos, 2600, Sala 120, Porto Alegre, RS, Brasil 90035 003. E-mail: wag_psico@yahoo.com.br
Independente da fonte estressora, diferentes estudos vêm demonstrando que o estresse impacta negativamente na saúde física e psicológica (Öhman, Bergdahl, Nyberg, \& Nilsson, 2007; Rod, Gronbaek, Schnohr, Prescott, \& Kristensen, 2009; Thoits, 2010). Tais estudos fornecem evidência de que a avaliação do estresse por meio de ferramentas validas e confiáveis é imprescindível para programas de prevenção, diagnóstico e intervenção em relação a esse problema.

Dentre as diferentes formas de avaliar o estresse, uma delas consiste em observar o grau no qual o indivíduo percebe como estressantes as diferentes situações ocorridas ao longo da sua vida (Lazarus \& Folkman, 1984). Tal perspectiva propõe que a presença de eventos de vida potencialmente estressores não caracteriza, necessariamente, o fenômeno do estresse (Lazarus, 1966, 1995; Lazarus \& Folkman, 1984). Para que um evento seja percebido como estressor, é necessária a ocorrência de dois processos mediadores entre o indivíduo e o ambiente: a avaliação cog- 
nitiva (cognitive appraisal), que determina em que medida o evento de vida é percebido como relevante/ameaçador; e as estratégias de coping, através do qual o indivíduo administra as demandas internas e externas frente ao evento percebido como estressor. O estresse ocorre, portanto, quando o indivíduo avalia que as demandas internas ou externas, excedem a sua capacidade para lidar com elas (Lazarus, 1995).

A Escala de Estresse Percebido (Perceived Stress Scale [PSS]; Cohen, Karmack, \& Mermelsteinm, 1983) é o instrumento mais utilizado para avaliar a percepção do estresse, tendo sido validada em mais de 20 países (Remor, 2006). A PSS avalia a percepção do indivíduo sobre o quão imprevisíveis e incontroláveis the parecem os eventos de vida experienciados no último mês, podendo ser utilizada na população geral com, no mínimo, nível de escolaridade equivalente ao ensino fundamental completo (Cohen \& Williamson, 1988). Além proporcionar uma avaliação subjetiva dos estresse, se destaca a brevidade do instrumento, o que favorece a sua aplicação em conjunto a outras medidas.

A PSS foi inicialmente desenvolvida com 14 itens (PSS-14, Cohen et al., 1983), sendo sete positivos e sete negativos. Uma análise de componentes principais, com rotação varimax, demonstrou a existência de dois componentes com autovalores $>1$ (3,6 e 2,2, respectivamente), refletindo os grupos de itens positivos e negativos (Cohen $\&$ Williamson, 1988). Posteriormente, foram produzidas versões reduzidas do instrumento, contendo 10 (PSS-10, seis positivos e quatro negativos) e quatro itens (PSS-4, dois positivos e dois negativos), sendo esta última utilizada apenas como instrumento de screening, em grandes levantamentos (Cohen \& Lichtensteins, 1990; Cohen \& Williamson, 1988).

A PSS-10 foi formada pelos itens da PSS-14 que apresentaram carga fatorial superior a 0,40 . Ao submeter a PSS-10 à uma análise de componentes principais, com rotação varimax, foram identificados dois componentes com autovalores $>1$ ( 3,4 e 1,4 , respectivamente), refletindo, novamente, os conjuntos de itens positivos e negativos do instrumento. Contudo, os autores sugerem que qualquer distinção feita entre os componentes ou fatores seria irrelevante, pois eles apenas refletiriam aspectos da redação dos itens (Cohen \& Williamson, 1988), onde os itens positivos e negativos tendem a se agruparem em dimensões distintas.

Apesar da orientação dos autores do instrumento em considerar a PSS-10 como um instrumento unidimensional, os resultados empíricos não têm sido consensuais. Alguns estudos (Campo-Arias, Bustos-Leiton, \& Romero-Chaparro, 2009; Cohen \& Williamson, 1988; Luft, Sanches, Mazo, \& Andrade, 2007; Mitchell, Crane, \& Kim, 2008) encontraram, a partir de técnicas exploratórias e confirmatórias, uma estrutura unifatorial para a escala, conforme postula a base teórica do instrumento (Cohen \& Williamsom, 1988), enquanto outros encontraram uma estrutura bifatorial (Leung, Lam, \& Chan, 2010; Roberti,
Harrington, \& Storch, 2010; Wongpakaran \& Wongpakaran, 2010). Nestes casos, os fatores são interpretados como estresse (itens positivos) e coping/controle (itens negativos), apresentando níveis de correlações variando entre $r=0,51$ e 0,65 .

No Brasil, as propriedades psicométricas da PSS-10 foram investigadas em dois estudos. Em uma amostra de 76 idosos, com idades entre 60 e 84 anos $(M=70,04$ anos, $D P=6,34)$, utilizando uma análise de componentes principais foi identificada uma estrutura unidimensional para a PSS-10. O componente retido explicou $42,5 \%$ da variância total dos escores, tendo consistência Alpha de Cronbach de 0,83 . Todos os itens apresentaram cargas componenciais superiores a 0,48 no componente retido (Luft et al., 2007).

Posteriormente, a PSS-10 foi avaliada em uma amostra de 793 professores universitários. Uma análise de componentes principais $(n=393)$ com rotação varimax, identificou a presença de dois componentes representando os conjuntos de itens positivos e negativos do instrumento (autovalores 4,62 e 1,05; Alpha de Cronbach de 0,83 e 0,77 , respectivamente). Em seguida, por meio de análises fatoriais confirmatórias $(n=400)$, foram comparados os índices de ajuste do modelo derivado da análise exploratória e de um modelo hierárquico, com dois fatores de primeira ordem e um fator de segunda ordem, denominado Estresse Percebido. O modelo hierárquico mostrou melhores índices de ajuste $(\mathrm{GFI}=0,94$; $\mathrm{AGFI}=0,90 ; \mathrm{RMR}=0,05$; CFI $=0,92)$ quando comparado ao modelo bifatorial $(\mathrm{GFI}=$ $0,91 ; \mathrm{AGFI}=0,88 ; \mathrm{RMR}=0,07 ; \mathrm{CFI}=0,88)$. A correlação entre fatores foi de 0,66 no estudo exploratório, e de 0,68 no estudo confirmatório. Os autores concluíram que os resultados sugerem uma estrutura unidimensional para a PSS-10, e que os dois fatores identificados nas análises fatoriais exploratória e confirmatória não deveriam ser interpretados separadamente (Reis, Hino, \& Rodriguez, 2010). Contudo, a descrição da estrutura unidimensional não é apresentada pelos autores.

Em síntese, os resultados de estudos internacionais e nacionais sobre a dimensionalidade da PSS-10 são contraditórios, sugerindo a adequação de ambos os modelos, uni e bifatorial. Uma possível explicação para as divergências encontradas em estudos anteriores sobre a dimensionalidade da EPP-10 diz respeito aos métodos de extração e retenção de fatores que deram origem a estrutura bifatorial da PSS-10 (Cohen \& Williamson, 1988; Reis et al., 2010). A análise de componentes principais é uma técnica de redução de dados, sendo desaconselhada para a identificação de fatores comuns, ou de traços latentes (Costello \& Osborne, 2005). Ainda, a utilização do critério Kaiser (autovalores maiores que 1) para retenção de fatores comuns tende a superestimar o número dimensões interpretadas (Horn, 1965; Humphreys \& Montanelli, 1975; Patil, Singh, Mishra, \& Donovan, 2008). É provável que a utilização de um critério conservador (e.g. análise paralela) para a retenção de fatores comuns conduza à interpretação de apenas um fator para o instrumento. 
Considerando os aspectos anteriormente mencionados, o presente estudo tem por objetivo investigar a dimensionalidade da PSS-10, com a finalidade de fornecer novas evidências de validade da PSS-10 no contexto brasileiro. Esse estudo é oriundo de um projeto maior, que teve por objetivo avaliar os níveis de estresse em uma amostra de professores de escolas públicas e particulares do ensino fundamental e médio. Entretanto, considerando o propósito do presente estudo, as características ocupacionais dos participantes não serão analisadas e discutidas.

\section{Método}

\section{Participantes}

Participou deste estudo uma amostra por conveniência de 517 professores do ensino fundamental e médio, de instituições públicas e privadas, da cidade de Campina Grande, Paraíba, Brasil. Destes 66,3\% são do sexo feminino, $54,9 \%$ casados e $50,5 \%$ possuem remuneração entre um e três salários mínimos. A idade dos participantes variou entre 18 e 65 anos $(M=36,52 ; D P=10,46)$.

\section{Procedimentos e Considerações Éticas}

O presente estudo foi realizado após parecer favorável do Comitê de Ética em Pesquisa da Universidade Estadual da Paraíba (UEPB; protocolo $n^{\circ}$. 0374.0.133.000-07). A coleta de dados seguiu as orientações éticas fornecidas pela Resolução 196/96 do Conselho Nacional de Saúde.

Antes de iniciar a coleta de dados, foi solicitado e recebido o consentimento da equipe diretiva das escolas e das secretarias municipais e estaduais de educação. Após estes procedimentos, foram agendados encontros com os professores para a apresentação dos objetivos do estudo. Todos os participantes responderam ao termo de consentimento livre e esclarecido (TCLE).

\section{Instrumentos}

Escala de Estresse Percebido (PSS-10). O instrumento é composto por 10 itens, sendo seis positivos e quatro negativos, respondidos em uma escala tipo Likert de frequência, variando de Nunca (0) à Sempre (4) (Cohen et al., 1983). A versão utilizada no presente estudo foi traduzida e adaptada para o português brasileiro por Luft et al. (2007).

Questionário Sociodemográfico. Esse instrumento visou levantar informações tais como idade, sexo, estado civil, grau de escolaridade, carga-horária, nível de ensino, dentre outros.

\section{Análise dos Dados}

Considerando o nível de mensuração ordinal das variáveis e a violação do pressuposto de normalidade multivariada dos dados (Mardia $=149,040, p \leq 0,05$; Mardia, 1970), foi conduzida uma análise fatorial exploratória robusta a partir da matriz de correlações policóricas dos itens da PSS-10 (Holgado-Tello, Chacón-Moscoso, Barbero-García, \& Vila-Abad, 2010; Muthén \& Kaplan,
1985), com método de extração minimum rank factor analysis (MRFA; Shapiro \& ten Berge, 2002). O método de extração MRFA minimiza a variância comum residual no processo de extração dos fatores, e possibilita a interpretação da proporção da variância comum explicada pelos fatores retidos (Lorenzo-Seva \& Ferrando, 2006).

Com a finalidade de evitar a superestimação de fatores comuns, adotou-se como método de retenção e interpretação dos fatores na análise fatorial exploratória a análise paralela (Horn, 1965). Este procedimento consiste em comparar os autovalores (ou a porção da variância total explicada) dos fatores extraídos da matriz de dados observados com a média de autovalores (ou da porção de variância total explicada) extraídos de uma série de matrizes geradas aleatoriamente, com o mesmo número de variáveis, casos observados, tipo de matriz de covariância, e método de extração de fatores (Buja \& Eyuboglu, 1992). São interpretados apenas os fatores comuns com valor explicativo superior aos fatores aleatórios. Foram geradas 500 matrizes de correlação policóricas pelo método de permutação aleatória dos valores observados. Este procedimento oferece estimativas mais fidedignas quando a distribuição amostral observada e populacional é não-normal (Timmerman \& Lorenzo-Seva, 2011). Estudos de simulação têm indicado que o uso conjunto do critério da análise paralela, da análise de matrizes de correlação policóricas e do método de extração MRFA apresenta melhor desempenho na recuperação do número de fatores da matriz populacional quando comparado com os métodos tradicionais (Timmerman \& Lorenzo-Seva, 2011). As análises foram conduzidas com o software Factor (v. 8.02; Lorenzo-Seva \& Ferrando, 2006).

\section{Resultados}

A matriz de correlações policóricas mostrou-se adequada para a extração de fatores, tendo os índices Kaiser-Meyer-Olkin $(\mathrm{KMO})=0,84$ e o teste de esfericidade de Bartlett $=743,2(45) ; p<0,001$. Pelo método de extração MRFA foram identificados dois fatores com autovalores $>1$ (3,08 e 1,16, respectivamente). Contudo, a análise paralela demonstrou que apenas um dos fatores comuns superou a porção de variância total explicada quando comparado à média dos fatores aleatórios (Figura 1). $\mathrm{O}$ fator retido (autovalor $=3,08$ ) explicou $67 \%$ da variância comum dos escores. As cargas fatoriais dos itens da PSS10 e a consistência interna da escala são apresentadas na Tabela 1.

Resultados praticamente idênticos foram obtidos com o método de extração de componentes principais e da análise paralela, sendo os autovalores observados 3,67 e 1,15 e os autovalores aleatórios 1,22 e 1,16 para o primeiro e segundo componente, respectivamente. O percentil 95 para os autovalores aleatórios foram iguais a 1,27 e 1,20 para o primeiro e segundo componente, respectivamente. Optou-se por reportar esses resultados para minimizar a hipótese de viés metodológico no presente estudo. 
Machado, W. L., Damásio, B. F., Borsa, J. L. \& Silva, J. P. (2014). Dimensionalidade da Escala de Estresse Percebido (Perceived Stress Scale, PSS-10) em uma Amostra de Professores.

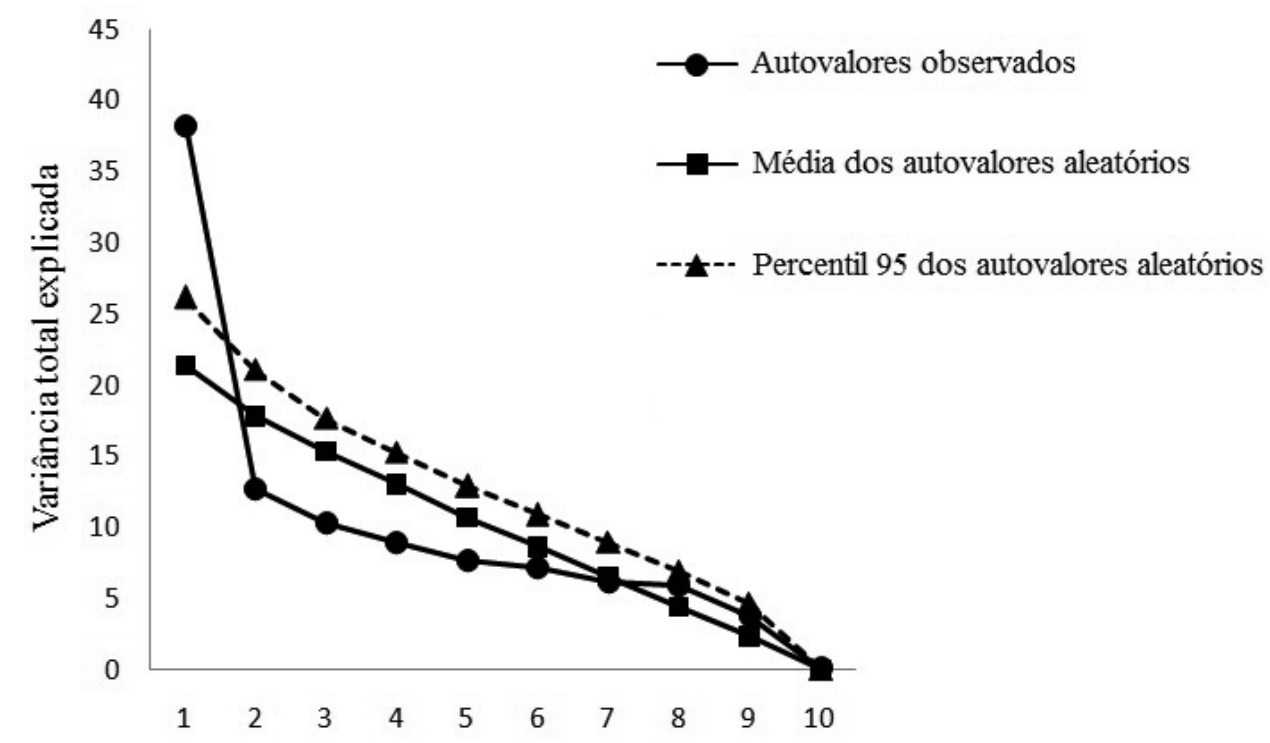

Figura 1. Análise paralela pelo método de permutação aleatória dos valores observados.

Tabela 1

Cargas Fatoriais dos Itens da PSS-10

Item (conteúdo abreviado)

Cargas Fatoriais

01 (aborrecido com algo inesperado)

0,43

02 (incapaz de controlar coisas importantes na vida)

0,63

03 (nervoso ou estressado)

0,42

04 (confiante em lidar com problemas pessoais)

0,58

05 (sentiu que as coisas aconteceram como esperado)

0,42

06 (não conseguiria lidar com todos os afazeres)

0,55

07 (controlar irritações na sua vida)

0,37

08 (todos os aspectos da vida sob controle)

0,44

09 (bravo devido a coisas fora de seu controle)

0,51

10 (sentiu os problemas se acumularem a ponto de não conseguir resolvê-los)

0,56

Média (Desvio-padrão)

$15,36(5,20)$

Alpha de Cronbach $(\alpha)$

0,80

\section{Discussão}

As análises fatoriais exploratórias, utilizando matriz de correlações policóricas, método de retenção do minimum rank factor analysis e o critério de retenção fatorial da análise paralela identificaram uma estrutura unidimensional para a PSS-10, em concordância com estudos prévios (Campo-Arias et al., 2009; Cohen \& Williamson, 1988; Luft et al., 2007; Mitchell et al., 2008). Este resultado era esperado, tendo em vista estudos prévios que demonstra- vam que todos os itens da PSS-10 apresentavam cargas componenciais iguais ou superiores a 0,40 quando retido apenas um componente (Cohen \& Williamson, 1988; Luft et al., 2007). Quando os itens da PSS-10 são separados em dois fatores, estes apresentam correlações de 0,51 a 0,69 , o que sugere uma moderada covariância entre ambas as dimensões (Reis et al., 2010; Roberti et al., 2010; Wongpakaran \& Wongpakaran, 2010).

O fator retido apresentou adequada consistência interna, e todos os itens da PSS-10 apresentaram cargas fatoriais 
de adequada magnitude $(\geq 0,37)$. Desta forma, o segundo fator identificado em estudos prévios parece refletir uma dimensão inconsistente, relativa à redação dos itens (positivos e negativos), não superando a proporção de variância explicada esperada ao acaso (Horn, 1965; Humphreys \& Montanelli, 1975; Patil et al., 2008). É importante salientar que os vieses de resposta a itens positivos e negativos são amplamente conhecidos na Psicologia (Ibrahim, 2001). Em alguns casos, a construção de instrumentos com itens positivos e negativos conduzem a vieses de respostas, repercutindo em soluções fatoriais oriundas de artefatos metodológicos, aparentemente sem sentido teórico (Marsh, 1996). Este parece ser o caso da PSS-10, em que os itens positivos e negativos da escala refletem comportamentos pertencentes a um mesmo continuum de estresse percebido (Cohen \& Williamson, 1998).

Conforme mencionado anteriormente, a PSS-10 é um instrumento breve que apresenta características psicométricas adequadas, podendo trazer vantagens em relação a instrumentos mais extensos. Tais vantagens são mais explícitas principalmente em situações de avaliação, quando se faz necessário a aplicação de muitos instrumentos, como em condições de pesquisa, por exemplo. Vale ressaltar, contudo, que a PSS-10 não se constitui um instrumento diagnóstico de estresse ocupacional, ou de avaliação de estresse relacionado a qualquer outro contexto. A PSS-10 é uma medida genérica de níveis de estresse percebido, de maneira que não são avaliadas as fontes causadoras de estresse. Caso o interesse seja avaliar o estresse ocupacional (i.e., os níveis de estresse relacionados especificamente ao trabalho), se deve buscar medidas específicas para esse fim.

\section{Considerações Finais}

O objetivo do presente estudo foi investigar a estrutura fatorial da Escala de Estresse Percebido, em sua versão de 10 itens (PSS-10), em uma amostra de professores de ensino fundamental e médio. Para atingir estes objetivos, utilizou-se de técnicas estatísticas robustas, com base nas características de distribuição e no nível de mensuração das variáveis (ordinal), com vistas à apresentação de resultados confiáveis. Os resultados da análise de fatores comuns e da análise paralela permitiram identificar a dimensionalidade da PSS-10, apontando uma solução unifatorial como a mais representativa dos dados. Entretanto, é importante salientar que a dimensionalidade da PSS-10 não é um consenso na literatura (Leung et al., 2010; Reis et al., 2010; Roberti et al., 2010; Wongpakaran \& Wongpakaran, 2010).

Desse modo, são sugeridas novas pesquisas que tenham como objetivo explorar a dimensionalidade da PSS-10 em diferentes populações, visto que o presente estudo utilizou uma amostra ocupacional específica, de uma região geográfica particular. Da mesma forma, recomenda-se o uso da análise paralela como critério de retenção de componentes ou fatores comuns em estudos exploratórios. Esse procedimento permite uma maior precisão na interpretação de dimensões de instrumentos, assinalando aquelas com poder explicativo maiores que o esperado de forma aleatória, diminuindo a probabilidade de superestimação no número de fatores retidos (Buja \& Eyuboglu, 1992; Horn, 1965; Humphreys \& Montanelli, 1975; Timmerman \& Lorenzo-Seva, 2011).

\section{Referências}

Buja, A., \& Eyuboglu, N. (1992). Remarks on parallel analysis. Multivariate Behavioral Research, 27(4), 509-540.

Campo-Arias, A., Bustos-Leiton, G. J., \& Romero-Chaparro, A. (2009). Internal consistency and dimensionality of the perceived stress scale (PSS-10 and PSS-14) in a sample of female university students in Bogotá, Colombia. Aquichán, 9(3), 271-280.

Codo, W. (1999). Educação: Carinho e trabalho. Petrópolis, RJ: Vozes.

Cohen, S., Karmack, T., \& Mermelsteinm, R. (1983). A global measure of perceived stress. Journal of Health and Social Behavior, 24(4), 385-396.

Cohen, S., \& Lichtenstein, E. (1990). Perceived stress, quitting smoking, and smoking relapse. Health Psychology, 9(4), 466-478.

Cohen, S., \& Williamsom, G. M. (1988). Perceived stress in a probability sample of United States. In S. Spacapan \& S. Oskamp (Eds.), The Social Psychology of Health: Claremont Symposium on applied social psychology. Newbury Park, CA: Sage.

Costello, A. B., \& Osborne, J. (2005, July). Best practices in exploratory factor analysis: Four recommendations for getting the most from your analysis. Practical Assessment Research \& Evaluation, 10(7). Retrieved December 07, 2011, from http://pareonline.net/getvn.asp?v=10\&n=7

Doménech Betoret, F., \& Gómez Artiga, A. (2010). Barriers perceived by teachers at work, coping strategies, self-efficacy and burnout. The Spanish Journal of Psychology, 13(2), 637-654. Retrieved from http://redalyc.uaemex.mx/redalyc/ pdf/172/17217376011.pdf

Holgado-Tello, F., Chacón-Moscoso, S., Barbero-García, I., \& Vila-Abad, E. (2010). Polychoric versus Pearson correlations in exploratory and confirmatory factor analysis of ordinal variables. Quality and Quantity, 44(1), 153-166.

Horn, J. L. (1965). A rationale and test for the number of factors in factor analysis. Psychometrika, 30(2), 179-185.

Humphreys, L. G., \& Montanelli, R. G. (1975). An examination of the parallel analysis criterion for determining the number of common factors. Multivariate Behavioral Research, 10(2), 193-205.

Ibrahim, A. M. (2001). Differential responding to positive and negative items: The case of a negative item in a questionnaire for course and faculty evaluation. Psychological Reports, 88(2), 497-500.

Kerr, R. A., Breen, J., Delaney, M., Kelly, C., \& Miller, K. (2011). A qualitative study of workplace stress and coping in secondary teachers in Ireland. Irish Journal of Applied Social Studies, 11(1). Retrieved from http://arrow.dit.ie/ijass/vol11/iss1/3

Kokkinos, C. M. (2007). Job stressors, personality and burnout in primary school teachers. British Journal of Educational Psychology, 77(1), 229-243.

Kyriacou, C. (2003). El estrés en la enseñanza. Revisión histórica y estado actual. In D. García-Villamisar \& T. Freixas-Guinjoan (Eds.), El estrés del professorado - una perspectiva internacional (pp. 39-59). Valencia, España: Promolibro. 
Machado, W. L., Damásio, B. F., Borsa, J. L. \& Silva, J. P. (2014). Dimensionalidade da Escala de Estresse Percebido (Perceived Stress Scale, PSS-10) em uma Amostra de Professores.

Lazarus, R.S. (1966). Psychological stress and the coping process. New York: McGraw-Hill.

Lazarus, R. S. (1995). Psychological stress in the workplace. In R. Crandall \& P. L. Perrewé (Eds.), Occupational stress: A handbook (pp. 3-14). Washington, DC: Taylor \& Francis.

Lazarus, R. S., \& Folkman, S. (1984). Stress. Appraisal, and Coping. Nova York: Springer.

Leung, D. Y. P., Lam, T., \& Chan, S. S. C. (2010). Three versions of Perceived Stress Scale: Validation in a sample of Chinese cardiac patients who smoke. Biomedicine Public Health, 10(1), 513.

Lorenzo-Seva, U., \& Ferrando, P. J. (2006). Factor: A computer program to fit the exploratory factor analysis model. Behavior Research Methods, 38(1), 88-91.

Luft, C. B., Sanches, S. O., Mazo, G. Z., \& Andrade, A. (2007). Versão brasileira da Escala de Estresse Percebido: Tradução e validação para idosos. Revista de Saúde Pública, 41(4), 606-615.

Mardia, K. V. (1970). Measures of multivariate skewnees and kurtosis with applications. Biometrika, 57(3), 519-530.

Marsh, H. (1996). Positive and negative global self-esteem: A substantively meaningful distinction or artifactors? Journal of Personality and Social Psychology, 70(4), 818-819.

Mitchell, A. M., Crane, P. A., \& Kim, Y. (2008). Perceived stress in survivors of suicide: Psychometric properties of the Perceived Stress Scale. Research in Nursing \& Health, 31(6), 576-585.

Montgomery, C., \& Rupp, A. A. (2005). A meta-analysis for exploring the diverse causes and effects of stress in teachers. Canadian Journal of Education, 28(3), 461-488.

Muthén, B., \& Kaplan D. (1985). A comparison of some methodologies for the factor analysis of non-normal Likert variables. British Journal of Mathematical and Statistical Psychology, 38, 171-189.

Öhman, L., Bergdahl, J., Nyberg, L., \& Nilsson, L. (2007). Longitudinal analysis of the relation between moderate long-term stress and health. Stress and Health, 23(2), 131-138.

Patil, V. H., Singh, S. N., Mishra, S., \& Donovan, T. (2008). Efficient theory development and factor retention criteria: A case for abandoning the 'eigenvalue greater than one' criterion. Journal of Business Research, 61(2), 162-170.

Reis, R. R., Hino, A. A. F., \& Rodriguez, C. R. (2010). Perceived Stress Scale: Reliability and validity study in Brazil. Journal of Health Psychology, 15(1), 107-114.

Remor, E. (2006). Psychometric properties of a European Spanish version of the Perceived Stress Scale (PSS). Spanish Journal of Psychology, 9(1), 86-93.

Roberti, J. W, Harrington, L. N., \& Storch, E. A. (2010). Further psychometric support for the 10-Item version of the Perceived Stress Scale. Journal of College Counseling, 9(2), 135-147.

Rod, N. H., Gronbaek, M., Schnohr, P., Prescott, E., \& Kristensen, T. S. (2009). Perceived stress as a risk factor for changes in health behavior and cardiac risk profile: A longitudinal study. Journal of Internal Medicine, 266(5), 467-475.

Shapiro, A., \& ten Berge, J. M. F. (2002). Statistical inference of minimum rank factor analysis. Psychometrika, 67(1), 79-94.

Thoits, P. A. (2010). Stress and Health: Major findings and policy implications. Journal of Health and Social Behavior, 51(1), 41-53.

Timmerman, M. E., \& Lorenzo-Seva, U. (2011). Dimensionality assessment of ordered polytomous itens with parallel analysis. Psychological Methods, 16(2), 209-220.
Wongpakaran, N., \& Wongpakaran, T. (2010, June). The Thai version of the PSS-10: An investigation of its psychometric properties. BiopsychoSocial Medicine, 12(4), 6, 2010, Retrieved December 07, 2011, from www.biomedcentral.com/ content/pdf/1751-0759-4-6.pdf

Yong, Z., \& Yue, Y. (2007). Causes for burnout among secondary and elementary school teachers and preventive strategies. Chinese Education and Society, 40(5), 78-85. 